\title{
Diabetic cachectic neuropathy: An uncommon neurological complication of diabetes
}

\author{
A Iyagba, MBBS, FWACP, FMCP; A Onwuchekwa, MBBS, FMCP \\ Neurology Unit, Medicine Department, University of Port Harcourt Teaching Hospital, Port Harcourt, Nigeria
}

Corresponding author: A Iyagba (amiyagba@gmail.com)

\begin{abstract}
A 40-year-old female patient with diabetes of 12 years' duration, with poor drug compliance, presented with a 4-month history of rapid progressive weight loss, burning sensations in the feet, abdominal swelling, and constipation with occasional episodes of epigastric pain. On examination, she was chronically ill-looking with a body mass index of $17.1 \mathrm{~kg} / \mathrm{m}^{2}$, grossly distended abdomen (initially mistaken for gravid abdomen). Blood pressure measurements in the supine and standing positions were $200 / 130 \mathrm{mmHg}$ and $180 / 100 \mathrm{mmHg}$, respectively. Neurological examination revealed stocking-pattern loss of pain, temperature, and light touch modalities. Vibration sensation was impaired up to the malleoli bilaterally, with impairment of joint position sense of both big toes. Random blood sugar level was $16.4 \mathrm{mmol} / \mathrm{L}$; glycosylated haemoglobin was $13.2 \%$ with a haematocrit of $33.0 \%$. Renal indices, uric acid, liver function tests and fasting lipid profile were all within normal limits. An abdominal ultrasound scan showed distended bowel loops. The vibration perception threshold average using biothesiometry was $27.3 \mathrm{mV}$.
\end{abstract}

S Afr Med J 2016;106(12):1190-1191. DOI:10.7196/SAMJ.2016.v106i12.11198

Diabetic patients can be affected by a wide variety of neurological complications which may involve the peripheral or autonomic nervous system, or both. These complications significantly impair the quality of life of patients, with impact on morbidity and mortality outcomes.

Diabetic cachectic neuropathy, also called diabetic neuropathic cachexia, is a very rare neurological complication of diabetes. ${ }^{[1]}$ It was first described by Ellenberg ${ }^{[2]}$ in 1934. It is associated with poor diabetic control that presents with profound unintentional weight loss associated with an acute symmetrical painful peripheral neuropathy without weakness. ${ }^{[3]}$

Pain is characteristically burning in nature, with predominant lower-limb involvement and allodynia. The disorder affects both type 1 and type 2 diabetics and occurs irrespective of the duration of diabetes. Depression and, in males, impotence appear to be common, although other autonomic features can be present.

Although a few case reports exist in the literature describing this condition, to the best of our knowledge, there has been no such report from our practice environment. This article serves to describe a very rare neurological complication of diabetes that highlights our diagnostic and management experience.

\section{Case report}

A 40-year-old female patient with diabetes of 12 years' duration, with poor drug compliance and clinic follow-up, presented to us at the accident and emergency department of the University of Port Harcourt Teaching Hospital with a 4-month history of rapid progressive weight loss, burning sensations in the feet, abdominal swelling, and constipation with occasional episodes of epigastric pain. These were associated with generalised body weakness, tiredness, and postural dizziness with occasional episodes of falling. She also admitted to florid, itching, offensive vaginal discharge. In addition, she had frothy urine, intermittent episodes of retrosternal chest pain, palpitations, and symptoms of hyperglycaemia. Her obstetric history was significant: two previous stillbirths and one second-trimester intrauterine fetal death. She had a strong family history of diabetes. She had been amennorrhoeic for about 5 months.

On examination, she was chronically ill-looking with a body mass index of $17.1 \mathrm{~kg} / \mathrm{m}^{2}$, grossly distended abdomen (initially mistaken for gravid abdomen). She had a tachycardia of $128 \mathrm{bpm}$; blood pressure measurements in the supine and standing positions were 200/130 $\mathrm{mmHg}$ and $180 / 100 \mathrm{mmHg}$, respectively. Her apex beat was not displaced but she had a third heart sound with gallop rhythm. There were reduced breath sounds in both lung bases. Neurological examination revealed stocking-pattern loss of pain, temperature and light touch modalities. Vibration sensation was impaired up to the malleoli bilaterally, with impairment of joint position sense of both big toes.

Her random blood sugar level was $16.4 \mathrm{mmol} / \mathrm{L}$; glycosylated haemoglobin was $13.2 \%$ with a haematocrit of $33.0 \%$. Renal indices, uric acid, liver function tests and fasting lipid profile were all within normal limits. Her urine was pale yellow and turbid. Urine microscopy showed $2-3$ pus cells/high power field with yeast and epithelial cells. Urine culture yielded scanty growth of Staphylococcus aureus (sensitive to cefuroxime, ceftriazone and perfloxacin) and moderate growth of Candida albicans. An abdominal ultrasound scan showed distended bowel loops. Her liver, spleen and kidneys were not enlarged. There was no ascites. Pregnancy test was negative. Electrocardiogram showed tachycardia, left atrial enlargement and left ventricular hypertrophy. Chest radiography showed cardiomegaly and unfolded aorta in keeping with hypertensive cardiovascular changes. A biothesiometer was used to determine her vibration perception threshold. This was obtained by taking the average of three readings $(\mathrm{R})$ obtained by placing the probe at the right toe (R1), left toe (R2) and again at the right toe (R3). The values obtained were $27 \mathrm{mV}, 28 \mathrm{mV}$ and $27 \mathrm{mV}$, respectively. The vibration perception threshold average was $27.3 \mathrm{mV}$.

Management consisted of optimising glycaemic control with regular insulin and blood pressure control with antihypertensive agents (lisinopril and amlodipine, both $10 \mathrm{mg}$ daily). She also received 
atorvastatin $10 \mathrm{mg}$ daily, clopidogrel $75 \mathrm{mg}$ daily and nystatin vaginal pessaries nocte (6 doses). Medication was initially carbamazepine controlled-release formulation $200 \mathrm{mg}$ twice daily for her neuropathic pain. This had little effect and was discontinued. She, however, obtained rapid relief and amelioration of her neuropathic lower-limb pain with the introduction of pregabalin at $75 \mathrm{mg}$ twice daily.

\section{Discussion}

Our patient had complications involving both her peripheral and autonomic nervous system. Our initial consideration, because of her distended abdomen, was that of a diabetic patient presenting with distal symmetrical peripheral neuropathy in pregnancy. However, abdominal distension was just one of the many features of autonomic neuropathy that she manifested. The others were constipation, tiredness and postural dizziness. Distended loops of bowel on abdominal ultrasonography are in keeping with autonomic neuropathy of the gastrointestinal tract. The marked postural drop in blood pressure $(20 / 30 \mathrm{mmHg})$ is a feature of cardiovascular autonomic neuropathy. The presence of autonomic symptoms is poorly prognostic in these patients.

The response of her neuropathic pain to pregabalin was dramatic. Pregabalin has been recommended as the first-line agent for the treatment of diabetic peripheral neuropathic pain in most guidelines. ${ }^{[4,5]}$
The San Antonio Consensus Criteria ${ }^{[6]}$ are commonly used to define diabetic neuropathy for research purposes, and recommend at least one measurement in five different categories for the diagnosis of diabetic neuropathy. For clinical neuropathy, the guidelines require symptoms and signs, or one of these with abnormal testing, or autonomic testing. Subclinical neuropathy is identified by abnormal testing only. Biothesiometry is an easy and objectively quantifiable means of diagnosing neuropathy in diabetic patients. A vibration perception threshold average of $\geq 25 \mathrm{mV}$ is diagnostic of neuropathy. We were unable to perform nerve conduction studies, considered the gold standard for neuropathy diagnosis, because of lack of availability.

1. Knopp M, Rajabally YA. Common and less common peripheral nerve disorders associated with diabetes. Curr Diab Rev 2012;8(3):229-236. http://dx.doi.org/10.2174/157339912800564034

2. Ellenberg M. Diabetic neuropathic cachexia. Diabetes 1974;23:418-423. http://dx.doi.org/10.2337/diab.23.5.418

3. Knopp M, Srikantha M, Rajabally YA. Insulin neuritis and diabetic cachectic neuropathy: A review. Curr Diabetes Rev 2013;9(3):267-274. http://dx.doi.org/10.2174/1573399811309030007

4. Attala N, Cruccua G, Barona R, et al. EFNS guidelines on the pharmacological treatment of neuropathic pain: 2010 revision. Eur J Neurol 2010;17(9):1113-1123. http://dx.doi.org/10.1111/j.14681331.2010.02999.x

5. Moulin DE, Clark AJ, Gilron I, et al. Pharmacological management of chronic neuropathic pain: Consensus statement and guidelines from the Canadian Pain Society. Pain Res Manage 2012;12(1):13-21. http://dx.doi.org/10.1155/2007/730785

6. American Diabetes Association-American Academy of Neurology: Report and recommendations of the San Antonio Conference on Diabetic Neuropathy (Consensus Statement). Diabetes Care 1988;11(7):592-597. http://dx.doi.org/10.2337/diacare.11.7.592

Accepted 28 June 2016. 